\title{
A Solution of the Cosmological Constant, Using Multiverse Version of Penrose CCC Cosmology, and Enhanced Quantization Compared
}

\author{
Andrew Beckwith \\ Physics Department, College of Physics, Chongqing University, Chongqing, China \\ Email: rwill9955b@gmai.com
}

How to cite this paper: Beckwith, A. (2021) A Solution of the Cosmological Constant, Using Multiverse Version of Penrose CCC Cosmology, and Enhanced Quantization Compared. Journal of High Energy Physics, Gravitation and Cosmology, 7, 559-571. https://doi.org/10.4236/jhepgc.2021.72032

Received: February 27, 2021

Accepted: April 18, 2021

Published: April 21, 2021

Copyright $\odot 2021$ by author(s) and Scientific Research Publishing Inc. This work is licensed under the Creative Commons Attribution International License (CC BY 4.0). http://creativecommons.org/licenses/by/4.0/

\begin{abstract}
We reduplicate the Book "Dark Energy" by M. Li, X.-D. Li, and Y. Wang, zero-point energy calculation with an unexpected "length" added to the "width" of a graviton wavefunction just prior to the entrance of "gravitons" to a small region of space-time prior to a nonsingular start to the universe. We compare this to a solution which worked out using Klauder Enhanced quantization, for the same given problem. The solution of the first Cosmological Constant problem relies upon the geometry of the multiverse generalization of CCC cosmology which is explained in this paper. The second solution used involves Klauder enhanced quantization. We look at energy given by our methods and compare and contrast it with the negative energy of the Rosen model for a mini sub-universe and estimate GW frequencies.
\end{abstract}

\section{Keywords}

Minimum Scale Factor, Cosmological Constant, Space-Time Bubble, Bouncing Cosmologies

\section{Introduction}

We bring up this study due to the general failure of field theory methods to obtain a working solution to the Cosmological Constant problem. One of the reasons for this work is that Quintessence studies involving evolution of the Cosmological Constant over time have routinely failed in match-ups with Early supernova data. The working hypothesis is that if Dark Energy is commensurate with the Cosmological Constant, that the Cosmological Constant, and Dark energy would be the same from the start of inflation. The traditional model of inflation also involves due to the Penrose Singularity theorems [1] a singular 
point for initial expansion of the Universe. Our study clearly initiates a Cosmological constant which is invariant over time. We digress from using the Penrose Singularity theorem [1] in terms of a Cosmic bounce [2] style nonsingular initial bubble of space-time for reason we state in the manuscript. The importance we have to our work is that we are convinced that the Cosmological Constant [3] [4] was set initially for conditions in the interior of a nucleated space-time bubble, with a wavefunction style calculation for collapsing infill into the space-time bubble with that wavefunction of Dark energy [5] [6] to be at a minimum 10 to the 30 power times Planck length. We conclude that Polarization states for obtaining early universe high-frequency gravitational waves detected and analyzed may be more favorable for higher-dimensional generalizations of our Dark Energy generating procedures, with Klauder Enhanced Quantization [7] [8] limited to $3+1$ Geometry whereas our Vacuum energy [9] [10] calculation may be amendable to higher frequency calculation incantations. While considering [11]-[19] issues, in particular [19] gives us future goals to consider. As an example, could multiverse CCC allow incorporating [19] with [13]?

\section{Methods}

We will first start off with the redone calculation as to the Vacuum energy as given in [1] and how we rescale them to be in sync as to the observed experimental value for vacuum energy which is of the present era. This methodology is consistent with the Zero-point energy calculation, we start off with the following as given by [11]

$$
\begin{aligned}
& \frac{1}{2} \cdot \sum_{i} \omega_{i} \equiv V(\text { volume }) \cdot \int_{0}^{\hat{\lambda}} \sqrt{k^{2}+m^{2}} \frac{k^{2} \mathrm{~d} k}{4 \pi^{2}} \approx \frac{\hat{\lambda}^{4}}{16 \pi^{2}} \\
& \underset{\hat{\lambda}=M_{\text {Planck }}}{\longrightarrow} \rho_{\text {boson }} \approx 2 \times 10^{71} \mathrm{GeV}^{4} \approx 10^{119} \cdot\left(\rho_{D E}=\frac{\Lambda}{8 \pi G}\right)
\end{aligned}
$$

In stating this we have to consider that $\rho_{D E}=\frac{\Lambda}{8 \pi G} \approx \hbar \cdot \frac{(2 \pi)^{4}}{\lambda_{D E}^{4}}$, so then that the equation we have to consider is a wavelength $\lambda_{D E} \approx 10^{30} \ell_{\text {Planck }}$ which is about $10^{30}$ times a Plank length radius of a space-time bubble [13] as a nonsingular expansion point for Cosmology, at the start of inflation with the space-time bubble of about a Plank length radius in size. Having said that, how do we get having the Penrose multiverse condition in this problem, for $\lambda_{D E} \approx 10^{30} \ell_{\text {Planck }}$ just before the near singularity?

\subsection{Looking Now at the Modification of the Penrose CCC (Cosmology)}

We now outline the generalization for Penrose CCC (Cosmology) just before inflation which we state we are extending Penrose's suggestion of cyclic universes, black hole evaporation, and the embedding structure our universe is contained within, this multiverse has $\mathrm{BHs}$ and may resolve what appears to be an impossible dichotomy. The following is largely from [13] and has serious relevance to 
the final part of the conclusion. That there are $N$ universes undergoing Penrose "infinite expansion" (Penrose) [18] contained in a mega universe structure. Furthermore, each of the $N$ universes has black hole evaporation, with Hawking radiation from decaying black holes. If each of the $\mathrm{N}$ universes is defined by a partition function, called $\left\{\Xi_{i}\right\}_{i=N}^{i=1}$, then there exists an information ensemble of mixed minimum information correlated about $10^{7}-10^{8}$ bits of information per partition function in the set $\left.\left\{\Xi_{i}\right\}_{i=N}^{i=1}\right|_{\text {before }}$, so minimum information is conserved between a set of partition functions per universe [13]

$$
\left.\left.\left\{\Xi_{i}\right\}_{i=N}^{i=1}\right|_{\text {before }} \equiv\left\{\Xi_{i}\right\}_{i=N}^{i=1}\right|_{\text {after }}
$$

However, there is non-uniqueness of information put into partition function $\left\{\Xi_{i}\right\}_{i \equiv N}^{i \equiv 1}$. Also Hawking radiation from black holes is collated via a strange attractor collection in the mega universe structure to form a new inflationary regime for each of the $N$ universes represented Our idea is to use what is known as CCC cosmology [13] [18], which can be thought of as the following. First. Have a big bang (initial expansion) for the universe which is represented by $\left\{\Xi_{i}\right\}_{i \equiv N}^{i \equiv 1}$. Verification of this mega structure compression and expansion of information with stated non-uniqueness of information placed in each of the $N$ universes favors ergodic mixing of initial values for each of $N$ universes expanding from a singularity beginning. The $n_{f}$ stated value, will be using $(\mathrm{Ng}$, 2008) $S_{\text {entropy }} \sim n_{f}$. [20]. How to tie in this energy expression, as in Equation (12) will be to look at the formation of a nontrivial gravitational measure as a new big bang for each of the $N$ universes as by $n\left(E_{i}\right)$ the density of states at energy $E_{i}$ for partition function [13] [14].

$$
\left\{\Xi_{i}\right\}_{i=1}^{i \equiv N} \propto\left\{\int_{0}^{\infty} \mathrm{d} E_{i} \cdot n\left(E_{i}\right) \cdot \mathrm{e}^{-E_{i}}\right\}_{i \equiv 1}^{i \equiv N} .
$$

Each of $E_{i}$ identified with Equation (3) above, are with the iteration for $N$ universes [13], and [18] (Penrose, 2006) Then the following holds, by asserting the following claim to the universe, as a mixed state, with black holes playing a major part, i.e.

CLAIM 1

See the below [13] representation of mixing for assorted $\mathrm{N}$ partition function per CCC cycle

$$
\left.\left.\frac{1}{N} \cdot \sum_{j=1}^{N} \Xi_{j}\right|_{j \text { before nucleation regime }} \stackrel{\text { vacuum nucleation tranfer }}{\longrightarrow} \Xi_{i}\right|_{i \text { fixed after nucleation regime }}
$$

For $N$ number of universes, with each $\left.\Xi_{j}\right|_{j \text { before nucleation regime }}$ for $j=1$ to $N$ being the partition function of each universe just before the blend into the RHS of Equation (4) above for our present universe. Also, each independent universes as given by $\left.\Xi_{j}\right|_{j \text { before nucleation regime }}$ is constructed by the absorption of one to ten million black holes taking in energy, i.e. (Penrose) [18]. Furthermore, the main point is done in [13] in terms of general ergodic mixing [21] [22] [23]. 
Claim 2

$$
\left.\left.\Xi_{j}\right|_{j \text { before nucleation regime }} \approx \sum_{k=1}^{\text {Max }} \tilde{\Xi}_{k}\right|_{\text {black holes jth universe }}
$$

What is done in Claim 1 and 2 [13] is to come up as to how a multi dimensional representation of black hole physics enables continual mixing of spacetime [13] [18] [22] [24] largely as a way to avoid the Anthropic principle [13] [25], as to a preferred set of initial conditions.

\subsection{Looking at the Modification of the Penrose CCC (Cosmology)}

We argue this modification is mandated by having the initial $\mathrm{DE}$ wavefunction set as

$$
\lambda_{D E} \approx 10^{30} \ell_{\text {Planck }}
$$

After having set this in place we will be comparing this method to the Klauder argument.

\subsection{Looking at Klauder Enhanced Quantization for Cosmological Constant}

We use the Padmanabhan $1^{\text {st }}$ integral [26] of the form, with the third entry of Equation (1) having a Ricci scalar defined via [27] [28] and usually the curvature $\aleph \quad$ [13] set as extremely small, with the general relativity [13] [28] action of

$$
\begin{aligned}
& S_{1}=\frac{1}{2 \kappa} \int \sqrt{-g} \cdot \mathrm{d}^{4} x \cdot(\Re-2 \Lambda) \\
& \&-g=-\operatorname{det} g_{u v} \\
& \& \Re=6 \cdot\left(\left(\frac{\ddot{a}}{a}\right)+\left(\frac{\dot{a}}{a}\right)^{2}+\frac{\aleph}{a^{2}}\right)
\end{aligned}
$$

\subsection{Next for the Idea from Klauder}

We are going to go to page 78 by Klauder [8] [13] of what he calls on page 78 a restricted Quantum action principle which he writes as: $S_{2}$ where we write a 1-1 equivalence as in [8] [13], which is

$$
S_{2}=\int_{0}^{T} \mathrm{~d} t \cdot\left[p(t) \dot{q}(t)-H_{N}(p(t), q(t))\right] \approx S_{1}=\frac{1}{2 \kappa} \cdot \int \sqrt{-g} \cdot \mathrm{d}^{4} x \cdot(\Re-2 \Lambda)
$$

Our assumption is that $\Lambda$ is a constant [13], hence we assume then use the approximation, from [8], and [13]

$$
\begin{aligned}
& \frac{p_{0}^{2}}{2}=\frac{p_{0}^{2}(N)}{2}+N ; \text { for } 0<N \leq \infty \text { and } q=q_{0} \pm p_{0} t \\
& V_{N}(x)=0 ; \text { for } 0<x<1 \\
& V_{N}(x)=N \text {; otherwise } \\
& H_{N}(p(t), q(t))=\frac{p_{0}^{2}}{2}+\frac{(\hbar \cdot \pi)^{2}}{2}+N ; \text { for } 0<N \leq \infty
\end{aligned}
$$

Our innovation is to then equate $q=q_{0} \pm p_{0} t \sim \phi$ and to assume small time step values. Then $[13]$ 


$$
\Lambda \approx \frac{-\left[\frac{V_{0}}{3 \gamma-1}+2 N+\frac{\gamma \cdot(3 \gamma-1)}{8 \pi G \cdot \tilde{t}^{2}}\right]}{\frac{1}{\kappa} \int \sqrt{-g} \cdot \mathrm{d}^{3} x}+\left.\left(6 \cdot\left(\frac{\ddot{a}}{a}+\left(\frac{\dot{a}}{a}\right)^{2}\right)\right)\right|_{t=\tilde{t}}
$$

If we assume that Equation (10) was formed within a cosmic bubble of space-time [12]

$$
\left(\frac{\dot{a}}{a}\right)^{2}=H_{\text {bounce-cos }}^{2}=\frac{8 \pi}{3 M_{P}^{2}} \cdot\left(\rho-\frac{\rho^{2}}{2|\sigma|}\right)
$$

Here, we have that $\rho$ is a space-time density function, whereas $\sigma$ is the tension of a space-time bubble presumably of the order of a Planck radius. Also within the bubble of space-time $\left(\frac{\ddot{a}}{a}\right) \approx \varepsilon^{+} \simeq 0$ and $a(t)=a_{\min } t^{\gamma}$ at the surface of the space-time bubble. Hence

$$
\Lambda \approx \frac{-\left[\frac{V_{0}}{3 \gamma-1}+2 \tilde{N}+\frac{\gamma(3 \gamma-1)}{8 \pi G t_{\text {Planck }}}\right]}{\frac{1}{\kappa} \int \sqrt{-g} \mathrm{~d}^{3} x}+c_{1} \frac{16 \pi}{M_{P}^{2}} \cdot\left(\rho-\frac{\rho^{2}}{2|\sigma|}\right)
$$

$c_{1}$ is to be determined, whereas the inflaton [13] [26].

$$
\phi=\sqrt{\frac{\gamma}{4 \pi G}} \ln \left\{\sqrt{\frac{8 \pi G V_{0}}{\gamma(3 \gamma-1)}} \cdot t\right\}
$$

And what we will use the "inflaton potential" we write as [13] [26]

$$
V=V_{0} \cdot\left\{\sqrt{\frac{8 \pi G V_{0}}{\gamma(3 \gamma-1)}} \cdot t\right\}^{\sqrt{\frac{\gamma}{4 \pi G}}-\sqrt{\frac{8 \pi G}{\gamma}}}
$$

Also

$$
\rho \approx \frac{\dot{\phi}^{2}}{2}+V(\phi) \equiv \frac{\gamma}{8 \pi G} \cdot t^{2}+V_{0} \cdot\left\{\sqrt{\frac{8 \pi G V_{0}}{\gamma(3 \gamma-1)}} \cdot t\right\}^{\sqrt{\frac{\gamma}{4 \pi G}}-\sqrt{\frac{8 \pi G}{\gamma}}}
$$

\section{Results: Comparing Equation (1) Implications with Equation (12)}

The significance of the procedure is that with tweaking we may be seeing the actual realization of classical gravity as an Eikonal Approximation to quantum theory [29] [30] as what was brought up by Horowitz and Oron in a highly nonstandard way as seen in reference [30] which gives a different interpretation to Equation (15) above. The term we refer to as Eikonal approximation to a quantum state has the form of the following decomposition. From Powell and Crasemann, [31] we have the decomposition of Geometric style decomposition of the optical wave equation of

$$
\nabla^{2} \psi-\frac{1}{v_{\text {Velocity }}^{2}} \cdot \frac{\mathrm{d}^{2} \psi}{\mathrm{d} t^{2}}=0
$$


whereas we use the common $\lambda \cdot \omega=2 \pi v_{\text {velocity }}$, and if we substitute in an angular frequency dependence of $\mathrm{e}^{-i \cdot \omega \cdot t}$ in $\psi$, we get from (16) that if $\lambda=h / p$ and $p=\sqrt{2 m \cdot(E-V(\text { potential }))}$, then we have a transformation of Equation (17) to Equation (18)

$$
\nabla^{2} \psi+\frac{4 \pi^{2}}{\lambda^{2}} \cdot \psi=0
$$

Lead to a Schrodinger equation of the form given by

$$
\nabla^{2} \psi+\frac{8 \pi^{2}}{h^{2}} \cdot(E-V(\text { potential })) \cdot \psi=0
$$

Equation (17) has [32]

$$
\psi=\sum_{\ell=0}^{\infty} \sum_{m=-\ell}^{\ell}\left(a_{\ell m} j_{\ell}(k r)+b_{\ell m} y_{\ell}(k r)\right) \cdot Y_{\ell}^{m}(\theta, \phi)
$$

where we could get away with making a substitution of [31]

$$
k=\frac{2 \pi}{\lambda}=\frac{2 \pi}{h} \cdot(E-V(\text { potential }))^{1 / 2}
$$

where the first and second Bessel Equation solutions [32] are of the form

$$
\begin{gathered}
j_{\ell}(k r=x)=(-x)^{\ell}\left(\frac{1}{x} \frac{\mathrm{d}}{\mathrm{d} x}\right)^{\ell} \frac{\sin x}{x} \\
y_{\ell}(k r=x)=-(-x)^{\ell}\left(\frac{1}{x} \frac{\mathrm{d}}{\mathrm{d} x}\right)^{\ell} \frac{\cos x}{x}
\end{gathered}
$$

Then we have via making use of Kieffer's result of variance of the energy [33] which is

$$
\Delta E=\frac{\sqrt{2 \tilde{n}+1}}{\lambda} \approx \frac{h \sqrt{2 \tilde{n}+1} \cdot(E-V(\text { potential }))^{1 / 2}}{2 \pi} \equiv \hbar \sqrt{2 \tilde{n}+1} \cdot \sqrt{\frac{V_{\text {volume }}}{8 \pi G}} \cdot t
$$

We get via [33] on page 239 of this reference, Equation (23), after we use (23), then

$$
\Delta E \Delta t \approx \sqrt{2 \tilde{n}+1} \cdot \sqrt{\frac{V_{\text {volume }}}{8 \pi G}} \cdot t \cdot \Delta t \cdot \hbar \approx \hbar
$$

If so, then we have that if so by use of [34] since this is also applicable to black holes

$$
\begin{aligned}
& t \propto t_{\text {Planck }} \propto \Delta t \Rightarrow \Delta E \Delta t \approx \sqrt{2 \tilde{n}+1} \cdot \sqrt{\frac{V_{\text {volume }}}{8 \pi G}} \cdot(\Delta t)^{2} \cdot \hbar \propto \hbar \\
& \Rightarrow(\Delta t)^{2} \propto \frac{1}{\sqrt{2 \tilde{n}+1}} \cdot \sqrt{\frac{8 \pi G}{V_{\text {volume }}}}
\end{aligned}
$$

Using Planck units, what this means is that if $G=\hbar=\ell_{\text {Planck }}=k_{B}=1=$ Planck time

$$
(\Delta t)^{2} \propto \frac{1}{\sqrt{2 \tilde{n}+1}} \cdot \sqrt{\frac{8 \pi G}{V_{\text {volume }}}} \equiv \sqrt{\frac{8 \pi}{2 \tilde{n}+1}} \cdot\left(t_{\text {Planck }}\right)^{2}
$$

If $\tilde{n}=$ quantum number, then for $\Delta t$ being proportional to $t_{\text {Planck }} \propto \Delta t$, we 
have definite restrictions on $\tilde{n}$ = quantum number , i.e. of the type given by

$$
\sqrt{\frac{8 \pi}{2 \tilde{n}+1}} \leq 1
$$

whereas the Kieffer Wavefunction commensurate as to Equation (23) for a quantum Dust universe initially is given as [33] on page239 of this reference

$$
\psi_{\tilde{n}, \lambda}(t, r) \equiv \frac{1}{\sqrt{2 \pi}} \cdot \frac{\tilde{n} ! \cdot(2 \lambda)^{\tilde{n}+1 / 2}}{\sqrt{(2 \tilde{n}) !}} \cdot\left[\frac{1}{(\lambda+i \cdot t+i \cdot r)^{\tilde{n}+1}}-\frac{1}{(\lambda+i \cdot t-i \cdot r)^{\tilde{n}+1}}\right]
$$

Here the time $t$ would be proportional to Planck time, and $r$ would be proportional to Planck length, whereas we set

$$
\lambda \approx \sqrt{\frac{8 \pi G}{V_{\text {volume }} \hbar^{2} t^{2}}} \underset{G=\hbar=\ell_{\text {Planck }}=k_{B}=1}{\longrightarrow} \sqrt{\frac{8 \pi}{t^{2}}} \equiv \frac{\sqrt{8 \pi}}{t}
$$

Then a preliminary emergent space-time wavefunction would take the form of

$$
\begin{aligned}
& \psi_{\tilde{n}, \lambda}(\Delta t, r) \\
& \equiv \frac{1}{\sqrt{2 \pi}} \cdot \frac{\tilde{n} ! \cdot\left(2 \cdot \sqrt{8 \pi} \cdot(\Delta t)^{-1}\right)^{\tilde{n}+1 / 2}}{\sqrt{(2 \tilde{n}) !}} \cdot\left[\frac{1}{\left(\sqrt{8 \pi} \cdot(\Delta t)^{-1}+i \cdot \Delta t+i \cdot r\right)^{\tilde{n}+1}}\right. \\
& \left.-\frac{1}{\left(\sqrt{8 \pi} \cdot(\Delta t)^{-1}+i \cdot \Delta t-i \cdot r\right)^{\tilde{n}+1}}\right]
\end{aligned}
$$

Just at the surface of the bubble of space-time, with $t_{\text {Planck }} \propto \Delta t$, and $r \propto \ell_{\text {Planck }}$

This is from a section, page 239 of the $3^{\text {rd }}$ edition of Kieffer's book, as to a quantum theory of collapsing dust shells, and is a way to use what is referred to as what Isham in 1984 referred to as "group quantization", as se need to remember that we are assuming $G=\hbar=\ell_{\text {Planck }}=k_{B}=t_{\text {Planck }}=1$. Having said that, let us look at Equation (1) and Equation (12) again.

\subsection{We Compare Equation (1) and Equation (12) with the above New Information}

$$
\begin{aligned}
& \Lambda \approx \frac{-\left[\frac{V_{0}}{3 \gamma-1}+2 \tilde{N}+\frac{\gamma(3 \gamma-1)}{8 \pi G t_{\text {Planck }}}\right]}{\frac{1}{\kappa} \int \sqrt{-g} \mathrm{~d}^{3} x}+c_{1} \frac{16 \pi}{M_{P}^{2}} \cdot\left(\rho-\frac{\rho^{2}}{2|\sigma|}\right) \\
& \Leftrightarrow \rho_{D E}=\left.\left.\frac{\Lambda}{8 \pi G} \Leftrightarrow \Lambda \approx \hbar \cdot 8 \pi G \cdot \frac{(2 \pi)^{4}}{\lambda_{D E}^{4}}\right|_{\lambda_{D E} \approx 10^{30} \ell_{\text {Planck }}} \equiv 8 \pi \cdot \frac{(2 \pi)^{4}}{\lambda_{D E}^{4}}\right|_{\lambda_{D E} \approx 10^{30}}
\end{aligned}
$$

To compare these two values we can state that within the bubble, that just before the bubble boundary, we have $\tilde{N}=0$, hence we look at, initially

$$
\Lambda \approx \frac{-\left[\frac{V_{0}}{3 \gamma-1}+\frac{\gamma(3 \gamma-1)}{8 \pi G t_{\text {Planck }}}\right]}{\frac{1}{\kappa} \int \sqrt{-g} \mathrm{~d}^{3} x}+c_{1} \frac{16 \pi}{M_{P}^{2}} \cdot\left(\rho-\frac{\rho^{2}}{2|\sigma|}\right)
$$




$$
\left.\left.\Leftrightarrow \Lambda \approx \hbar \cdot 8 \pi G \cdot \frac{(2 \pi)^{4}}{\lambda_{D E}^{4}}\right|_{\lambda_{D E} \approx 10^{30} \ell_{\text {Planck }}} \equiv 8 \pi \cdot \frac{(2 \pi)^{4}}{\lambda_{D E}^{4}}\right|_{\lambda_{D E} \approx 10^{30}}
$$

i.e. we pick $C_{1}$ so that the two are equivalent in value, Note this is just before a very large value set to $\tilde{N}$ at the boundary of the initial space-time bubble. Hence we also would be looking at

$$
\begin{aligned}
\Lambda & \approx \gamma \cdot t^{2}+8 \pi V_{0} \cdot\left\{\sqrt{\frac{8 \pi V_{0}}{\gamma(3 \gamma-1)}} \cdot t\right\}^{\sqrt{\frac{\gamma}{4 \pi}}}-\left.\sqrt{\frac{8 \pi}{\gamma}}\right|_{t \approx t_{\text {Planck }}=1} \\
& \approx \frac{-\left[\frac{V_{0}}{3 \gamma-1}+\frac{\gamma(3 \gamma-1)}{8 \pi}\right]}{\frac{1}{\kappa} \int \sqrt{-g} \mathrm{~d}^{3} x}+c_{1} \frac{16 \pi}{M_{P}^{2}} \cdot\left(\rho-\frac{\rho^{2}}{2|\sigma|}\right) \\
& \left.\approx \hbar \cdot 8 \pi G \cdot \frac{(2 \pi)^{4}}{\lambda_{D E}^{4}}\right|_{\lambda_{D E} \approx 10^{30} \ell_{\text {Planck }}}=\left.8 \pi \cdot \frac{(2 \pi)^{4}}{\lambda_{D E}^{4}}\right|_{\lambda_{D E} \approx 10^{30}}
\end{aligned}
$$

In Equation (31) we will also make the following identification

$\left.\rho \approx \rho_{D E} \approx \hbar \cdot \frac{(2 \pi)^{4}}{\lambda_{D E}^{4}}\right|_{\lambda_{D E} \approx 10^{30}}$ i.e. just before the bubble, we will factor in a very large bubble tension, $\sigma$. Doing so would be to have an optimal $c_{1}$ value, and that this choice of $c_{1}$ would allow us to make the identification of the choice of wavefunction for our model which would according to the Equation (28) model have a quantum flavor, while at the same time in adherence be similar to the Equation (31) values of Dark Energy density.

\subsection{What We Obtain If We Model the Cosmological Constant This Way?}

First of all, as noted by Kieffer, the wavefunction in Equation (28) vanishes if $r=$ 0 . We avoid having a vanishing wavefunction by asserting that we will be making an evaluation of it in the neighborhood of a Planck Length. Secondly in making this assumption we are at least proceeding in a direction where we can ditch the Anthropic principle, which I consider a MAJOR blight on cosmology. I also too though this procedure as outline is experimentally verifiable, once we understand what may constitute embedding of Quantum Mechanics within a deterministic structure. The entire referral of this problem can be contrasted with the solution given in [19] by Rosen, which is in the mode of Quantum mechanics for a single particle in an isotropic universe.

The primary difference between the Rosen approach, [19] and what is done in this document is that the mass of a graviton, is proportional to the square of the Cosmological constant, an assumption which Rosen does not make. In addition the Rosen document assumes that "The situation is somewhat analogous to the quantum mechanics of a single particle as compared to quantum field theory" and assumes an isotropic background. 
We do not confine ourself to Isotropic backgrounds. We also do share with Rosen [19] a procedure as to rendition a linkage between classical to quantum mechanical treatment of our problem, but our work is more in tune with using what Powell and Craseman in their book on quantum mechanics used, [ ] which is similar to a limiting case of geometric optics.

I wish to thank Christian Corda as to alerting me to the Rosen article which is also applicable to a Dust Cosmology. I also wish to thank Fangyu Li, Wen Hao, and others for alerting me as to the crucial role of polarization. As it is, I presented part of this idea in Zeldovich 4, in the ICRANET on-line conferences (a small part of it) and found that the audience and the reviewers did not understand the Klauder treatment of the cosmological constant via Klauder Enhanced quantization.

Hence this expanded treatment whereas the Rosen treatment as Corda outlined is most effective for quantum black holes.

\subsection{Final Reference to High Frequency Gravitational Waves}

$$
\begin{aligned}
& \left(1+z_{\text {initial era }}\right) \equiv \frac{a_{\text {today }}}{a_{\text {initial era }}} \approx\left(\frac{\omega_{\text {Earth orbit }}}{\omega_{\text {initial era }}}\right)^{-1} \\
& \Rightarrow\left(1+z_{\text {initial era }}\right) \omega_{\text {Earth orbit }} \approx 10^{25} \omega_{\text {Earth orbit }} \approx \omega_{\text {initial era }}
\end{aligned}
$$

Equation (34) can be seen in [35] as well as [36]. Whereas we postulate that we specify an initial era frequency via dimensional analysis which is slightly modified by Maggiore for the speed of a graviton [35] whereas

$$
c(\text { light speed }) \approx \omega_{\text {initial era }} \cdot\left(\lambda_{\text {initial post bubble }}=\ell_{\text {Planck }}\right)
$$

and that dimensional comparison with initially having a temperature built up so as

$$
\Delta E \approx \hbar \omega_{\text {initial era }}
$$

where $T_{\text {universe }} \approx T_{\text {Plank temerature }}=1.22 \times 10^{19} \mathrm{GeV}$. If so then the Planck era temperature would be extremely high leading to a change in temperature from the Pre Planckian conditions to Planck era leading to

$$
\Delta E=\frac{d(\operatorname{dim})}{2} \cdot k_{B} \cdot T_{\text {universe }}
$$

In doing so, be assuming

$$
\omega_{\text {initial era }} \approx \frac{c}{\ell_{\text {planck }}} \leq 1.8549 \times 10^{43} \mathrm{~Hz}
$$

where we would be assuming $\omega_{\text {initial era }} \approx \frac{C}{\ell_{\text {planck }}} \leq 1.8549 \times 10^{43} \mathrm{~Hz}$ so then we would be looking at frequencies on Earth from gravitons of mass $m$ (graviton) less than of equal to

$$
\omega_{\text {Earth orbit }} \leq 10^{-25} \omega_{\text {initial era }}
$$

And this partly due to the transference of cosmological "information" as given in 
[13] for a phantom bounce type of construction. Further point that since we have that gravitons travel at nearly the speed of light [36], that gravitons are formed from the surface of a bubble of space-time up to the electroweak era that mass values of the order of $10^{-65}$ grams (rest mass of relic gravitons) would increase due to extremely high velocity would lead to enormous $\Delta E \approx \hbar \omega_{\text {initial era }}$ values per graviton, which would make the conflation of ultrahigh temperatures with gravitons traveling at nearly the speed of light as given in Equation (39) as compared with $\Delta E \approx \hbar \omega_{\text {initial era }}$.

We can in future work compare this with the Rosen [19] value of energy for a mini universe of (from a Schrodinger equation) with ground state mass of $m=\sqrt{\pi} M_{\text {Planck }}$ and an energy of

$$
E_{\hat{n}}=\frac{-G m^{5}}{2 \pi^{2} \hbar^{2} \widehat{n}^{2}}
$$

Our preliminary supposition is that Equation (40) could represent the initial energy of a Pre Planckian Universe and that Equation (37) be the thermal energy dumped in due to the use of Cyclic Conformal cosmology (maybe in multiverse form) so that if there is a build up of energy greater than Equation (40) due to thermal build up of temperature due to infall of matter-energy, we have a release of Gravitons in great number which would commence as a domain wall broke down about in the Planckian era with a temperature of the magnitude of Planck Energy for a volume of radius of the order of Plank Length. This will be investigated in detailed future calculations. All this should be in fidelity, in experimental limits to [37], as well as looking at ideas about Quantum tunneling we may gain from [38] as to understand the transition from Pre Plankian to Planckian physics [39] [40].

\section{Acknowledgements}

This work is supported in part by National Nature Science Foundation of China grant No. 11375279.

\section{Conflicts of Interest}

The author declares no conflicts of interest regarding the publication of this paper.

\section{References}

[1] Hawking, S. and Ellis, G.F.R. (1973) The Large Scale Structure of Space-Time. Cambridge University Press, Cambridge. https://doi.org/10.1017/CBO9780511524646

[2] Nojiri, S., et al. (2019) Nonsingular Bounce Cosmology from Lagrange Multiplier F(R) Gravity. Physical Review D, 100, Article ID: 084056. https://arxiv.org/abs/1910.03546 https://doi.org/10.1103/PhysRevD.100.084056

[3] Wang, Q.D., Zhu, Z. and Unruh, W.G. (2017) How the Huge Energy of Quantum 
Vacuum Gravitates to Drive the Slow Accelerating Expansion of the Universe. Physical Review D, 95, Article ID: 103504.

https://doi.org/10.1103/PhysRevD.95.103504

[4] Adler, R.J., Casey, B. and Jacob, O.C. (1995) Vacuum Catastrophe: An Elementary Exposition of the Cosmological Constant Problem. American Journal of Physics, 63, 620-626. https://doi.org/10.1119/1.17850

[5] Astier, P., et al. (2006) The Supernova Legacy Survey: Measurement of $\Omega_{\mathrm{M}}, \Omega_{\Lambda}$ and W from the First Year Data Set. Astronomy and Astrophysics, 447, 31-48. https://doi.org/10.1051/0004-6361:20054185

[6] Frieman, J.A., Turner, M.S. and Huterer, D. (2008) Dark Energy and the Accelerating Universe. Annual Review of Astronomy and Astrophysics, 46, 385-432. https://doi.org/10.1146/annurev.astro.46.060407.145243

[7] Klauder, J.R. (2017) Enhanced Quantization: The Right way to Quantize Everything. https://arxiv.org/abs/1702.04713

[8] Klauder, J.R. (2015) Enhanced Quantization: Particles, Fields \& Gravity. World Scientific, Singapore. https://doi.org/10.1142/9452

[9] Sola, J. (2013) Cosmological Constant and Vacuum Energy: Old and New Ideas. Journal of Physics. Conference Series, 453, Article ID: 012015.

https://arxiv.org/abs/1306.1527 https://doi.org/10.1088/1742-6596/453/1/012015

[10] Seminar, P., Duplantier, B. and Rivasseau, V. (2003) Poincaré Seminar 2002: Vacuum Energy-Renormalization. Progress in Mathematical Physics, Vol. 30. Birkhäuser Verlag, Basel. https://doi.org/10.1007/978-3-0348-8075-6

[11] Li, M., Li, X.-D., Wang, S. and Wang, Y. (2015) Dark Energy. Peking University Press, World Scientific, Singapore.

[12] Freeze, K., Brown, M. and Kinney, W. (2012) The Phantom Bounce, a New Proposal for an Oscillating Cosmology. In: Mercini-Houghton, L. and Vaas, R., Eds., The Arrows of Time, a Debate in Cosmology, Fundamental Theories in Physics, Volume 172, Springer Verlag, Heidelberg, 149-156.

https://doi.org/10.1007/978-3-642-23259-6 7

[13] Beckwith, A.W. (2021) Using "Enhanced Quantization" to Bound the Cosmological Constant, (for a Bound-On Graviton Mass), by Comparing Two Action Integrals (One Being from General Relativity) at the Start of Inflation. In: Sidharth, B.G., Murillo, J.C., Michelini, M. and Perea, C., Eds., Fundamental Physics and Physics Education Research, Springer Nature, Cham, 21-36.

https://doi.org/10.1007/978-3-030-52923-9 3

[14] Poplawski, N. (2011) Cosmological Constant from QCD Vacuum and Torsion. Annals of Physics (Berlin), 523, 291. https://doi.org/10.1002/andp.201000162

[15] Saunders, S. and Brown, H.R. (1991) The Philosophy of Vacuum. Clarendon Press, Oxford.

[16] Bass, S.D. (2015) Vacuum Energy and the Cosmological Constant. Modern Physics Letters A, 30, Article ID: 1540033. https://arxiv.org/abs/1503.05483

[17] Kieffer, K. (2012) Quantum Gravity. 3rd Edition, Oxford Science Publications, Oxford University Press, Oxford.

[18] Penrose, R. (2006) Before the Big Bang: An Outrageous New Perspective and Its Implications for Particle Physics. Proceedings of the EPAC 2006, Edinburgh, 26-30 June 2006, 2759-2762. 
[19] Rosen, N. (1993) Quantum Mechanics of a Miniuniverse. International Journal of Theoretical Physics, 32, 1435-1440. https://doi.org/10.1007/BF00675204

[20] Ng, Y.J. (2008) Spacetime Foam: From Entropy and Holography to Infinite Statistics and Nonlocality. Entropy, 10, 441-461. https://doi.org/10.3390/e10040441

[21] Halmos, P. (1956) Ergodic Theory. No. 3, Mathematical Society of Japan, Tokyo.

[22] Arnold, V.I. and Avez, A. (1968) Ergodic Problems of Classical Mechanics. W.A. Benjamin, Inc., San Francisco.

[23] Sarig, O. (2020) Lecture Notes on Ergodic Theory. http://www.weizmann.ac.il/math/sarigo/sites/math.sarigo/files/uploads/ergodicnote s.pdf

[24] Lectures by Maryam Mirzakhani Notes by Tony Feng. Introduction to Ergodic Theory. https://www.mit.edu/ fengt/ergodic theory.pdf

[25] Barrow, J.D. and Tipler, F.J. (1986) The Anthropic Cosmological Principle. Oxford University Press, Oxford.

[26] Padmanabhan, T. (2006) An Invitation to Astrophysics. World Scientific Series in Astronomy and Astrophysics: Volume 8, World Press Scientific, Singapore.

https://doi.org/10.1142/6010

[27] Compère, G. (2019) Advanced Lectures on General Relativity. https://doi.org/10.1007/978-3-030-04260-8

[28] Weinberg, S. (1972) Gravitation and Cosmology: Principles and Applications of the General Theory of Relativity. John Wiley and Sons, Cambridge.

[29] Lévy, M. and Sucher, J. (1969) Eikonal Approximation in Quantum Field Theory. Physical Review, 186, 1656. https://doi.org/10.1103/PhysRev.186.1656

[30] Horwitz, L. and Oron, O. (2006) Classical Gravity as an Eikonal Approximation to a Manifestly Lorentz Covariant Quantum Theory with Brownian Motion. In: Reimer, A., Ed., Quantum Gravity Research Trends, Nova Science Publishers, Inc., New York, Chapter 5, 143-190.

[31] Powell, J.L. and Crasemann, B. (1961) Quantum Mechanics. Addison-Wesley Company Inc., Reading.

[32] Arfken, G., Weber, H. and Harris, F. (2012) Mathematical Methods for Physicists, a Comprehensive Guide. 7th Edition, Academic Press, Elsevier, San Francisco.

[33] Kieffer, C. (2012) Quantum Gravity. Third Edition, Oxford Science Publications, Oxford University Press, Oxford.

[34] Kieffer, C. (2001) Path Integrals in Quantum Cosmology. In: Janke, W., et al., Eds., Fluctuating Paths and Fields, World Press Scientific, Singapore, 729-740. https://doi.org/10.1142/9789812811240 0062

[35] Maggiorie, M. (2008) Gravitational Waves, Volume 1, Theory and Experiment. Oxford University Press, New York.

[36] Landau, L.D. and Lifshitz, E.M. (1975) Classical Theory of Fields (Fourth Revised English ed.). Pergamon, Oxford.

[37] Misner, C., Thorne, K.S. and Wheeler, J.A. (1973) Gravitation. W.H. Freeman, San Francisco.

[38] Roy, D.K. (1993) A Quantum Measurement Approach to Tunnelling. World Press Scientific, Singapore.

[39] Vasileiou, V., Granot, J., Piran, T. and Amelino-Camelia, G. (2015) A Planck-Scale Limit on Spacetime Fuzziness and Stochastic Lorentz Invariance Violation. Nature 
Physics, 11, 344-346. https://doi.org/10.1038/nphys3270

[40] Perlman, E.S., Rappaport, S.A., Christensen, W.A., Jack Ng, Y., DeVore, J. and Pooley, D. (2014) New Constraints on Quantum Gravity from X-Ray and Gamma-Ray Observations. The Astrophysical Journal, 805, 10.

https://doi.org/10.1088/0004-637X/805/1/10 